\title{
ANTROPOLOGÍA
}

\section{EL SEÑALAMIENTO DE LA CRONICIDAD, LA TRANSFORMACIÓN EN EL ÁREA DE LO PÚBLICO Y LA INFLUENCIA DE LOS ENFERMEROS EN LA FORMACIÓN DE UN CUASI GRUPO}

Miret García, María Teresa', Bernalte Benazet, Alvaro², Gala León, Francisco Javier ${ }^{3}$, Lupiani Jiménez, María Mercedes ${ }^{4}$ ${ }^{1}$ Lda. en Geografía e Historia especialidad en Antropología Social. Máster en Antropología de la Salud. ${ }^{2}$ Ldo. en Antropología Social y Cultural, Doctor en Medicina. ${ }^{3}$ Ldo. en Psicología, Doctor en Medicina. ${ }^{4}$ Licenciada en Humanidades. Dra. en Medicina.

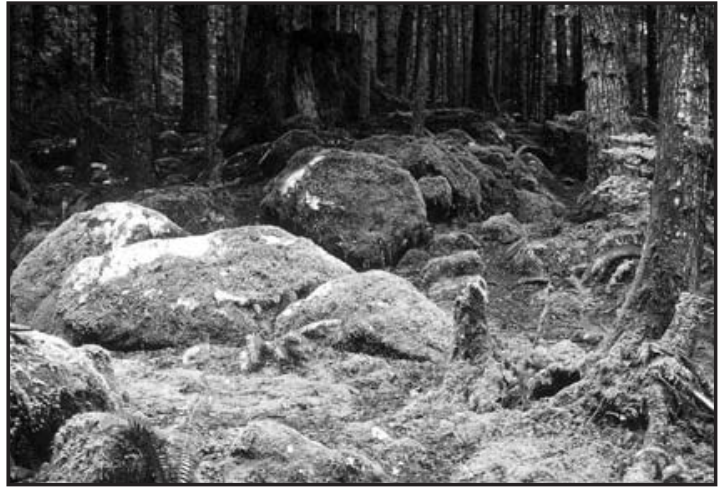

THE SIGNALING OF CHRONICITY, THE PUBLIC AREA TRANSFORMATION AND THE INFLUENCE OF THE NURSES IN THE FORMATION OF A QUASI-GROUP

\section{SUMMARY}

$\mathrm{F}$ Trom the 80's (XXth century) in the development of Primary Health Care in Spain and particularly in Andalusia, nurses' work has had a great influence, especially on the assistance to people with chronic diseases. The health education offered to these chronic patients, for them to achieve the necessary knowledge to tell healthy from unhealthy ways of life, has had an effect on what we could call the urban landscape, where we have detected the presence of these citizens who have made themselves visible in gardens, parks, walking, etc.

During more than one year we have shared their places of exercise in a small Mediterranean village, and by means of participant observation and verbal interaction we have obtained from these groups of people the how and the why of what is happening.

Key words: nursing care. Social impact of the nurses' work.

OS SINAIS DE CRONICIDADE, A TRANSFORMAÇÃO NA ÁREA DO PÚBLICO E DA INFLUÊNCIA NA FORMAÇÃO DOS ENFERMEIROS DE UM QUASE-GRUPO

\section{RESUMO}

partir da década de 80 (século XX), no
desenvolvimento da Atenção Primária de
Saúde na Espanha e, mais concretamente na Andaluzia, o trabalho dos enfermeiros tem tido uma grande influência, especialmente na atenção a pessoas que padecem de processos crônicos. A educação para a saúde efetuada nos pacientes crô- 
nicos, com o intuito de alcançar o nível de conhecimentos necessário para eleger entre as formas de vida saudável e não saudável, tem produzido uma influência no que podemos denominar de paisagem urbana, na qual temos observado a presença desses cidadãos, tornando-se visíveis, seja nos jardins, nos parques; a maioria deles em caminhadas, etc. Durante mais de um ano, temos compartilhado seus lugares de exercício em um pequeno vilarejo no Mediterrâneo, bem como através da observação participante e de interação verbal, temos obtido a partir destes grupos explicações sobre como e o porquê do que acontece.

Palavras-chave: Cuidados de enfermagem, Impacto social do trabalho de enfermagem.

\section{RESUMEN}

$\mathrm{A}$ partir de la década de los 80 (s. XX) en el desarrollo de la Atención Primaria de Salud en España y más concreto en Andalucía, el trabajo de la enfermera ha tenido una gran influencia, especialmente en la atención a las personas con procesos crónicos. La educación para la salud efectuada en los pacientes crónicos, cuyo objetivo era alcanzar el nivel de conocimientos necesario para elegir entre formas de vida sana e insana, ha producido una afectación en lo que podríamos llamar el paisaje urbano, donde hemos detectado la presencia de estos ciudadanos que se han hecho visibles, en los jardines, en los parques, la mayoría mediante caminatas, etc. Durante más de un año hemos compartido sus lugares de ejercicio en una pequeña aldea pequeña del mediterráneo, y a través de la observación participante y de la interacción verbal hemos obtenido de estos grupos de personas el cómo y el porqué de lo que sucede.

Palabras clave: Cuidados enfermeros. Impacto social del trabajo enfermero.

\section{INTRODUCCIÓN}

Debemos comentar que la presente etnografía ha sido realizada en un pequeño pueblo de la costa mediterránea española y cuyo nombre real no men- cionaremos, siguiendo una antigua tradición antropológica, así que lo denominaremos Micropolis.

Si nos centramos en el primer escalón asistencial de la medicina biologicista de nuestro país, la Atención Primaria de Salud, veremos que generalmente está representada a nivel formal - infraestructural por los Centros de Salud, y en ella, como sabemos desarrollan su actividad entre otros profesionales: enfermeros, médicos, trabajadores sociales, ,... etcétera.

En la práctica del enfermero de atención primaria también llamado enfermero de familia, se produce frecuentemente el ritual de tránsito de un cliente a enfermo crónico, y generalmente el ciudadano ya ha pasado por un prueba previa (rito), el denominado "señalamiento" o diagnóstico que es establecido por la única autoridad social facultada incluso para hacer morir a un ciudadano, el médico (Van Gennep, 1981; García, Velasco, et al., 1991).

Es decir un buen / mal día una persona que sale de su casa como un ciudadano de tantos, y al regresar de la consulta de su médico se da cuenta, de que ya no es aquella persona que fue, de pronto, es un enfermo y este término afecta a su identidad, a sus roles y a su estatus; pero cuando esta idea que parece rondar de forma inquietante, penetra o se introduce realmente en la mente, la vida y las actividades de la persona, es cuando un enfermero le toma de la mano cual padrino (entendido como actor principal en cualquier ritual) en el proceso de tránsito como enfermo crónico.

La idea primigenia del sujeto es la negación, cualquier excusa es buena para negar la realidad "científica", representada por el diagnóstico médico, pero en esa batalla su "partenaire" (el enfermero) no le va a acompañar, para el enfermero el paciente es una persona primero, y es alguien que puede tener una necesidades de cuidados que hay que cubrir, muchas veces no refiere siquiera al diagnóstico médico, habla incluso de diagnósticos enfermeros, lo que a muchos clientes les parece más una enumeración de... como llamarle... ¿cosas necesarias?... ¿necesidades?... En esa dinámica piensa la persona, pero yo estoy bien ... (duda) por qué necesito todas estas cosas, si trabajo, mantengo a mi familia, salgo con los amigos... ¿Por qué?. Es el enfermero quien armado de paciencia - si 
paciencia, muchas veces - va poco a poco situando a la persona en un contexto de "realidad" entendiendo esta como la realidad del enfermero, que sigue siendo percibida como irrealidad, por ahora, por "el paciente".

Tanto en la educación para la salud del paciente, tanto como en la parte de educación sobre su enfermedad la denominada educación sanitaria (Bernalte, Miret; 2003; Salleras; 1985), los estilos de vida que se le "exigen" a este nuevo hombre / mujer denominado crónico, tienen que ver generalmente con aspectos que van desde la nutrición al ejercicio físico, pasando por otras cuestiones; es en este aspecto del ejercicio físico donde estableceremos una realidad que parte de nuestra observación.

La presencia de personas de diferentes edades realizando ejercicio en el paseo marítimo de una Micrópolis, fue nuestro inicial estímulo y mediante técnicas de tipo cualitativo hemos realizado una investigación del paisaje humano descubierto y de algunas de sus causas que entendíamos podían estar relacionadas con los patrones de cambio de estilo de vida que se promociona entre los enfermos crónicos.

\section{MATERIAL Y MÉTODOS}

Realizamos inicialmente una búsqueda bibliográfica en la base de datos de CUIDEN y posteriormente en Medline, de los años 2003 a 2005 ambos incluidos, con las siguientes palabras clave: cuidados enfermeros e impacto social del trabajo enfermero, donde al no encontrar referencias ampliamos las palabras clave, a: paisaje urbano, paisaje, señalamiento, señalamiento de la cronicidad y cronicidad, y su expresión en inglés, y esto nos ha llevado a comprobar que ciertas referencias apenas tocan tangencialmente nuestra investigación, y en ningún caso directamente, aunque las incluimos en la bibliografía.

Nuestra investigación podemos definirla como dentro del paradigma cualitativo, epistemológicamente como constructivista, y metodológicamente hemos utilizado la etnografía, y nuestras técnicas son cualitativas.

Nuestro objetivo investigador, parte de una pregunta que uno de los autores se planteó, ¿qué hacen estas personas andando o corriendo por el paseo marítimo de la localidad?, serán acaso otros crónicos como yo, que intentan controlar su peso o disminuir los cúmulos de grasa ya existentes, y por ende cambiar su estilo de vida, o a diferencia de estos, son personas que simplemente se ejercitan para mejorar su condición física ("los valores que entraña la propia carrera, el cuerpo, la hazaña física o simplemente el bienestar..." Segalen; 1994:12).

El contexto ya mencionado es una pequeña localidad del sur mediterráneo, con un paseo marítimo que tiene el movimiento de personas más continuo de todas las calles y callejas de la mencionada, no es solo un balcón a la playa es punto de paso y de encuentro, con las terrazas de bares y restaurantes y sus bancos mirando el mar.

Las unidades de observación propuestas fueron tres, a saber:

A y B) Los dos tramos de paseo marítimo existente en Micrópolis, son punto de paso y de encuentro como ya mencionábamos, y lugar de ejercicio físico para muchas personas, hay que indicar que el paseo marítimo, padece una fractura de la continuidad debida a unos edificios públicos muy cerca del agua, y ello crea de hecho dos paseos marítimos; los dos tienen una cierta continuidad, el uno con un puerto deportivo y el otro con una serie de urbanizaciones situadas a pie de playa. El primero de ellos (a partir de ahora los denominaremos primer y segundo paseos), está delimitado en su longitud por una larga valla de obra de un edificio destinado a colonias veraniegas infantiles, es utilizado por los adolescentes para pasear con sus motocicletas, haciendo ruido y algún caballito que otro, comer pipas, beber alguna "cosilla", y fumarse algún que otro porro, siendo también utilizado como lugar de paso hacia el pueblo, por todos los habitantes del "pueblo marinero", es decir los apartamentos construidos alrededor del puerto deportivo, lo cual hace que existan por ejemplo unas variaciones de interés entre la época escolar, cuando las madres acompañan a sus hijos pequeños a la guardería y al colegio, sitos en el punto de fractura del paseo marítimo, y el período vacacional. El segundo paseo, más cercano al núcleo del pueblo, posee un frontal de bares, restaurantes y cafeterías, que a medida que se aleja del centro es substituido por grandes edificios de 
apartamentos, que también serán sustituidos a su vez por urbanizaciones de adosados, ...etcétera; también en él comer pipas y tirar sus cáscaras al suelo es una costumbre, así como colillas, papeles, y las "necesidades" de algunos animalitos, cuando no lo hacen en la arena de la playa, contigua al paseo, pero en esta zona es donde el Ayuntamiento utiliza casi todos sus medios de limpieza.

C. El quiosco de la prensa, que es un lugar de encuentro no solo por que esta tienda (que además es locutorio) es también un negocio que atrae a un público heterogéneo, sino por la "particular" simpatía de sus dueños que lo convierten en un centro de interacción entre individuos muy diferentes, interacciones donde los investigadores podían introducirse sin problema alguno, cuando no las generaban ellos mismos.

Las unidades de análisis definidas fueron cuatro:

- Los andadores y corredores como tales

- Los motivos de su actividad

- Sus interacciones en el paseo

- Sus conversaciones en el quiosco

Sobre la metodología utilizada en este trabajo de campo, diremos previamente a su descripción, que entendemos la investigación como... " acción, el mundo está ahí pero el mundo no viene a nosotros, no impacta, sino es a través de un abrir la ventana a él, los investigadores de la vida [que no somos otra cosa] debemos asomarnos a él, pero con una actitud de prestar atención, sólo esa atención inequívoca permite que penetre la información, ello es especialmente importante en el caso de los antropólogos que somos a la vez instrumento de recogida y elemento de procesamiento y análisis; todo ello parece fundamentar la gran reorientación de la práctica de nuestra disciplina realizada por Malinowski (Velasco;1999:131-153), en su propuesta sobre la aproximación a la "realidad" efectuada en "Los Argonautas del Pacífico Occidental" (Bernalte; 2004:94).

Nuestra elección de técnicas (consensuada entre los participantes) ha sido la siguiente:

* Observación participante, que procede de la reformulación realizada por Bronislaw Malinowski (1986:53-78), podríamos defi- nirla como una observación directa realizada por el etnógrafo, que implica una relación con los sujetos y objetos de estudio, que permite recoger no sólo la visión etic (la del etnógrafo) sino también la visión emic (de los "otros"), y sobre los contextos e interacciones que suceden en la institución o la comunidad que estudiamos, ha sido estudiada por diferentes autores como Taylor y Bogdam: "La observación participante... designa la investigación que involucra la interacción social entre el investigador y los informantes en el milieu de los últimos, y durante la cual se recogen datos de modo sistemático y no intrusivo" (1992: 31); Guasch: "La observación participante es uno de los modos de investigación que permite prestar mayor atención al punto de vista de los actores. Tal y como pretenden los clásicos, se trata de que el investigador se convierta el mismo en un nativo a través de la inmersión en la realidad social que analiza" (1970: 36); y otros: Del Rincón et al. (1995), Sebastián de Erice (1994).

En el planteamiento de la observación, y dado que en general las personas varían su forma natural de comportarse al sentirse observadas, hemos realizado un tipo de observación encubierta (Bernalte; Rev. Enf. Cient. 2000:214-5).

* Conversaciones informales, realizadas tanto en el quiosco como en el paseo (aunque prioritariamente en el primero) que nos permitían sin descubrir el objetivo 1 de la misma, obtener información sobre las causas de su presencia en el paseo marítimo (paseando, caminando, corriendo o en bicicleta).

* Asistencia a la consulta en el módulo de atención primaria existente en dicha localidad, para ver como se producía esa información o formación sobre estilos de vida saludables para enfermos crónicos, donde hemos de mencionar se incidía prioritariamente en algunos hábitos saludables, entre ellos el no fumar. 


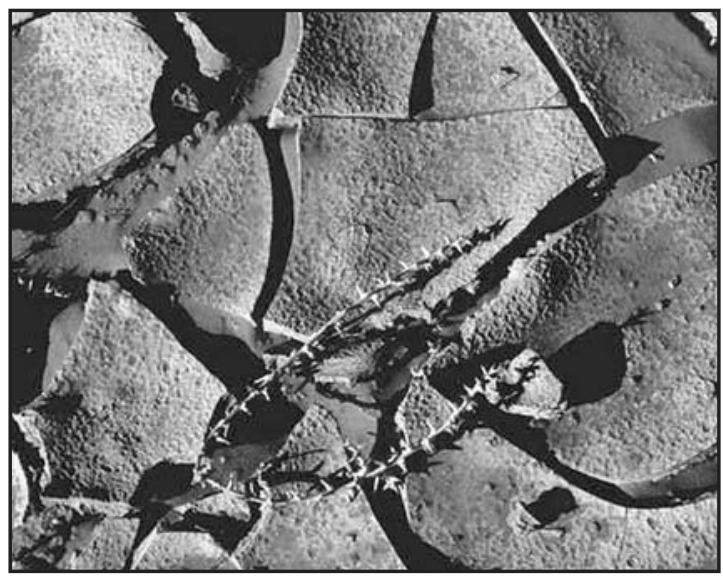

La guía de observación, creada por los antropólogos del grupo, fijaba la atención de los mismos en los siguientes aspectos:

- El proceso que había llevado allí a estas personas

- El objetivo de las mismas al realizar aquel ejercicio

- El tipo de ejercicio que realizaban y como lo hacían

- Algunas características de esas personas (edad, ropa, calzado, ...)

- Y el lugar donde realizaban el ejercicio

La observación se ha realizado durante 14 meses, abarcando inicialmente dos horarios: uno por la mañana y otro por la tarde, tanto en un paseo como en el otro inicialmente. En la primera fase de la observación nos situamos como uno más de los viandantes y corredores que atraviesan el primer paseo, realizando a lo largo de ocho meses una observación en dos horarios relacionados con la luz solar, uno del amanecer hasta las once horas y otro desde dos horas antes de decaer la luz del sol, el atardecer fue abandonado por la poca presencia de casos; en la siguiente fase de la observación nos situamos en el segundo paseo, y decididamente en horario matutino, durante 6 meses, aunque en el primer mes esporádicamente exploramos por la tarde el paseo.

La observación en el quiosco y las conversaciones informales, después de tres meses previos de introducirnos en el lugar, se han realizado a lo largo del mismo período.

\section{RESULTADOS}

Uno. El primer aspecto a reflejar es que la cronicidad de una enfermedad, supera la separación de lo privado de lo público.

Dos. La observación de las transformaciones conseguida por la escucha y las conversaciones informales de los clientes de tipo crónico, en el ámbito de lo público, nos impacta por denotar claramente la existencia de un antes y un después, en este momento se produce un cambio en los discursos, en los que se introducen:

- Afirmaciones sobre la salud en general.

- Comentarios sobre medidas dietéticas en particular.

- Afirmaciones sobre la importancia del estilo de vida.

- Comentarios sobre el ejercicio físico.

- Afirmaciones sobre las personas conocidas que deberían también adoptar cambios en su vida

- Otras.

Tres. Hemos observado varias actitudes con respecto al impacto que produce esta transformación en crónico, a saber:

- Primera. Una muy corriente es la de "invencible o irreductible" que refiere a la resistencia a aceptar que su situación ha cambiado y que por lo tanto debe tomar una serie de medidas versus el mantenimiento de su salud, generalmente va acompañada por una lucha constante con los miembros de su núcleo de convivencia o familiar y sobre todo con la cuidadora principal (si ésta es la mujer).

- Segunda. Otra es la de la cuidadora habitual que pasa a ser enferma crónica, en este caso la persona acostumbra a realizar algunos ajustes en su vida (sobre todo a nivel farmacológico, quizás son los más asumibles, y los que menos problemas producen al resto de la familia) pero de manera que no alteren apenas la vida de su entorno o núcleo de convivencia, es una actitud de "abnegación", se anteponen siempre las necesidades, gustos,... de los demás.

- Tercera. También existe la "aceptación" que parece traducida en derrota de la praxis de 
vida que uno ha llevado y que normalmente genera esa sensación de caída, un dejarse llevar por los especialistas: médico y enfermero con resignación, ese dejarse, comporta una actitud que lleva a veces a pequeñas rebeliones contra sus vigilantes, dado que no se ha producido un cambio profundo.

- Cuarta. La del "converso", que es la persona que acepta su nueva situación como un motivo para reorientar su atención sobre: si mismo, sus necesidades, sus objetivos y lo concreta en su cuerpo; en estos casos el individuo cual pez en el agua, realiza una transformación donde sus normas de vida son sustituidas por el decálogo de la vida sana, en cuanto a medidas higiénico - dietéticas, lo cual produce un impacto claro en su estilo de vida y que a veces afecta a su entorno próximo.

Cuatro. Los médicos y enfermeros no son conscientes de que sus consejos sobre el estilo de vida implican un cambo de costumbres y que este lo hemos observado en el paisaje urbano

Cinco. En la primera fase, en el primer paseo, pudimos observar en cuanto a las personas que lo utilizaban en el período del "amanecer", lo siguiente:

- Madres y alguna vez padres que llevan a los niños al colegio (en período escolar), trabajadores sobre todo varones, inmigrantes (sudamericanos y magrebíes en cuanto a su importancia numérica) la mayoría que van a sus quehaceres, sobre todo en la primera hora.

- Caminantes, sobre todo varones solos, también algunas mujeres en pequeños grupos de dos o tres personas. Entre las particularidades de estos, podemos mencionar cierta tendencia al saludo al cruzarse con otros caminadores, son personas que en su mayoría presentan una obesidad mórbida, existe también algún hiperglucémico y alguna persona con problemas circulatorios como las varices en los miembros inferiores; y sus edades oscilaban entre los cincuenta y los setenta años.

- Corredores, pocos, algún varón y alguna mujer; en su mayor parte son extranjeros (ingleses), lo realizan como deporte para mantenerse en forma, y sus edades varían, dado que excepto en el verano los corredores se sitúan entre los cuarenta y cinco y los sesenta y cinco, bajando el limite inferior muchísimo en los veranos, hasta los veinticinco. Estos, nunca saludan a los que caminan.

- Ciclistas, son muy pocos y su aparición es esporádica.

Seis. En la siguiente fase de la observación nos situamos en el segundo paseo, y decididamente en horario matutino; al amanecer existen en este mayor número de personas paseando, corriendo, caminando y en bicicleta que en el otro tramo del paseo, las características de los mismos:

- Algunos pescadores que ramonean sentados en las sillas de una terraza de un bar (cerrado por su puesto a esas horas), algún inmigrante sudamericano que se dedica a la limpieza de alguno de los muchos bares, y algunos camareros y camareras (magrebíes) que van al primero de sus quehaceres, que es abrir el establecimiento.

- Caminantes, existen algunos y su edad oscila entre los 45 y los 75 años, su número varía considerablemente en verano, por la incorporación numerosísima de veraneantes; entre los anuales podemos decir que existen los varones que caminan solos, y las mujeres en pequeños grupos, los primeros son más constantes , siendo las segundas las que faltan con una cierta frecuencia a esta cita diaria, entre los hombres, sus problemas son crónicos HTA y diabetes, y entre las mujeres predominan la obesidad, los problemas osteoarticulares, así como la HTA y la diabetes; dicho esto de pasada, entre los veraneantes, podemos distinguir: obesos e hipertensos.

- Corredores, apenas existen, algunos que combinan correr y caminar, y no continúan mucho tiempo, su edad tiene una gran dispersión desde 35 a 70 años, siendo la mayor parte de ellos ingleses, a parte de estas apariciones esporádicas, es en verano cuando su número se multiplica, bajando su media de edad, y situándose el limite inferior cercano a los 25 años, siendo su objetivo el estar en forma. 
- Ciclistas, existen pocos ciclistas, dos, y no son muy constantes, su edades oscilan entre 35 y 60 años, su preocupación es mantenerse en forma y no engordar.

\section{DISCUSIÓN}

En cuanto a la separación entre público y privado, de lo observado podemos afirmar que si bien es diagnosticada y tratada clínicamente por el médico en la privacidad de su despacho, y así mismo, es abordada en la intimidad de la consulta por el enfermero en cuanto a las necesidades individuales y la manera de afrontarlas, su propia existencia y afrontamiento la convierten en algo público, el enfermo crónico cambia generalmente sus costumbres, su estilo de vida y ello se determina y hace en parte en la fracción privada de la vida, pero también en la pública, así los cambios son perceptibles por las personas que lo rodean no solo en el núcleo familiar, sino en el ámbito laboral, en el del ocio, etcétera.

Esta afectación de la vida cotidiana implica como decíamos un cambio, generalmente, importante en el estilo de vida, como ejemplo: las personas con gran apetito pasan a "disfrutar" de dietas, es decir del control de los aportes: calóricos, proteicos,... ;así mismo, otras personas, muchas de ellas con una trayectoria de vida sedentaria, empiezan a salir a la calle vestidas, al parecer, de forma deportiva para realizar un ejercicio moderado que les ayude, en ese cambio del estilo de vida, y este es otro apunte a tomar en cuenta, dado que el ejercicio se realiza en gimnasios, clubes, pero prioritariamente se da en la calle, en los paseos, y en los jardines.

Si bien la categorización de las actitudes con respecto al impacto que produce esta transformación en crónico no pretende ser exhaustiva, diremos que entre estas posturas tan nítidas existen mezclas de las mismas, así como el paso de unas a otras. Esto que hemos recogido en el devenir de las conversaciones con los enfermos crónicos, procede del ámbito de lo privado, pero también puede observarse en el área de lo público, en el pueblecito objeto de este estudio. Y hemos podido aproximarnos a estas categorías por la utilización que de los espacios públicos como el paseo marítimo, hacen las personas que allí habitan a lo largo del año. Hemos obviado a los veraneantes aunque algunos de ellos por su perfil podrían formar parte.

El impacto en la geografía urbana, de la actuación de los enfermeros y los médicos de atención primaria, debido a sus consejos sobre el ejercicio físico moderado y los beneficios resultantes, es desconocido por los mismos, y de ello son ejemplo las dos partes del paseo marítimo de la pequeña población costera estudiada.

Algo que nos ha llamado la atención es que la mayoría de los caminantes no llevan ni ropa ni calzado adecuado, los tiempos de paseo son insuficientes e interrumpidos por paradas no justificables para el objetivo que pretenden; y que los más constantes pueden identificarse por que llevan - en general- la indumentaria más adecuada para realizar ejercicio físico.

\section{CONCLUSIONES}

La actuación del Servicio Andaluz por medio de sus programas de atención de crónicos está permitiendo explorar a muchas personas otro tipo de vida más saludable, a la cual muchos se lanzan como patos al agua, pero se puede observar:

- Faltan conocimientos de cómo se debe realizar el ejercicio, que tipo de calzado e indumentaria deberían utilizar para ello.

- Existen deserciones, personas que parecen no motivadas y abandonan, y otras que realizan períodos de ejercicio y períodos sin ejercicio.

- Existe un abandono o falta de tutela del proceso en cuanto las personas salen del centro de salud, creemos que esto no debería ser así, el proceso debería ser tutelado por los enfermeros de atención primaria de alguna forma.

- Debería estimularse la formación de cuasi grupos donde el apoyo mutuo estimulara e impidiera las deserciones.

\section{BIBLIOGRAFÍA}

- Andrews, J.; Manthorpe, J.; Watson, R. (2004) Involving older people in inmediate care. Journal of Advanced Nursing. 46(3): 303-310.

- Bernalte, A.; Miret, M. T. (2003). "Guía de educación para la salud desde la Mirada antropológica". Cádiz. Servicio de publicaciones de la Universidad de Cádiz.

- Bernalte, A. et al. (2000) "La observación una herramienta de enfermería que debe utilizarse siempre". : Rev. Enfermería Científica. Enero - Febrero. No 214 - 215. 
- Bernalte, A. (2004). "Etnografía de un centro de salud". Cádiz. Servicio de Publicaciones de la Universidad de Cádiz. - Bucnall, T. (2003) The clinical landscape of critical care: nurses' decision-making.Journal of Advanced Nursing 43(3): 310-319.

- Del Rincón, D.; Arnal, J. et al. (1995). "Técnicas de investigación en Ciencias Sociales". Madrid. Ed. Dykinson.

- García, J. L.; Velasco, H. et al. (1991). "Rituales y proceso social". Madrid. Ministerio de Cultura.

- García Mata, M Ángeles, (2005) "El final del final..." . Rev. Excel Enferm. Enero.

- Guasch, O. (1970) "La observación participante". (Cuadernos metodológicos $n^{\circ}$ 20) CIS, Madrid.

- Malinowski, B. (1986). "Els argonautes del Pacífic Occidental I". Barcelona. Edicions 62 / Diputació de Barcelona.

- Medina López, Ofelia Mercedes (20003) Encuentro con la cronicidad. Desarrollo Científ. Enferm. 2003 abr. 11(3):79-82.

- Mojsa, W.; Owlasiuk, A. Wiad Lek (2004) "Preparatory training of primary health care nurses for hanling health education regarding prophylaxis of circulatory disorders" Vo. 57 Suppl. 1, pp. 223-6.

- Nawafleh, H.; Francis, K; Chapman, Y. (2005) The influence of HIV/AIDS on the practice od primary care in Jordan: Rhetoric and reality. Internacional Journal of Nursing Practice. 11:200-205.

- Salleras, LL., (1985). "Educación Sanitaria. Principios,
Métodos y Aplicaciones”. Madrid. Ed. Díaz de Santos.

- Sanches, Luciane Maximiliano y Boemer, Magali Roseira (2002) O Convivo com a dor: um enfoque existencial. Rev Esc Enferm USP. dic. 36(4):386-393.

- Serra París, María y Comelles, Josep María. (1998) Acerca de la construcción sociocultural de la cronicidad. Rev. Trab Soc y Salud. mar. 29:315-323.

- Sebastián de Erice, J. (1994). "Erving Goffman. De la interacción focalizada al orden interaccional ". Madrid. CIS.

- Segalen, M. (1994). "El etnógrafo, el periodista y el médico". Granada.

- Taylor, S. J.; Bogdan, R. (1992). "Introducción a los métodos cualitativos de investigación. La búsqueda de significados". Ediciones Paidos. Barcelona.

- Van Gennep, A. (1981). "Les rites de passage". Paris. Ed. Picard.

- Vasconcelos Lira, Geison; Nations, Marilyn K. y Fontenelle Catrib, Ana María (2004) "Cronicidade e cuidados de saude: o que a antropologia de saúde tem a nos ensimar?. Contexto Enferm. ene-mar. 13(1):147-155

- Velasco, H. (1999). "Lecturas de Antropología social y cultural”. Madrid. UNED.

- Villagra de Ramírez, Liliana (1994) "Experiencia de enfermería y trabajo con familias en formación" Santafé de Bogotá D. C. Colombia. Ponencia II Congreso de actualización en enfermería.

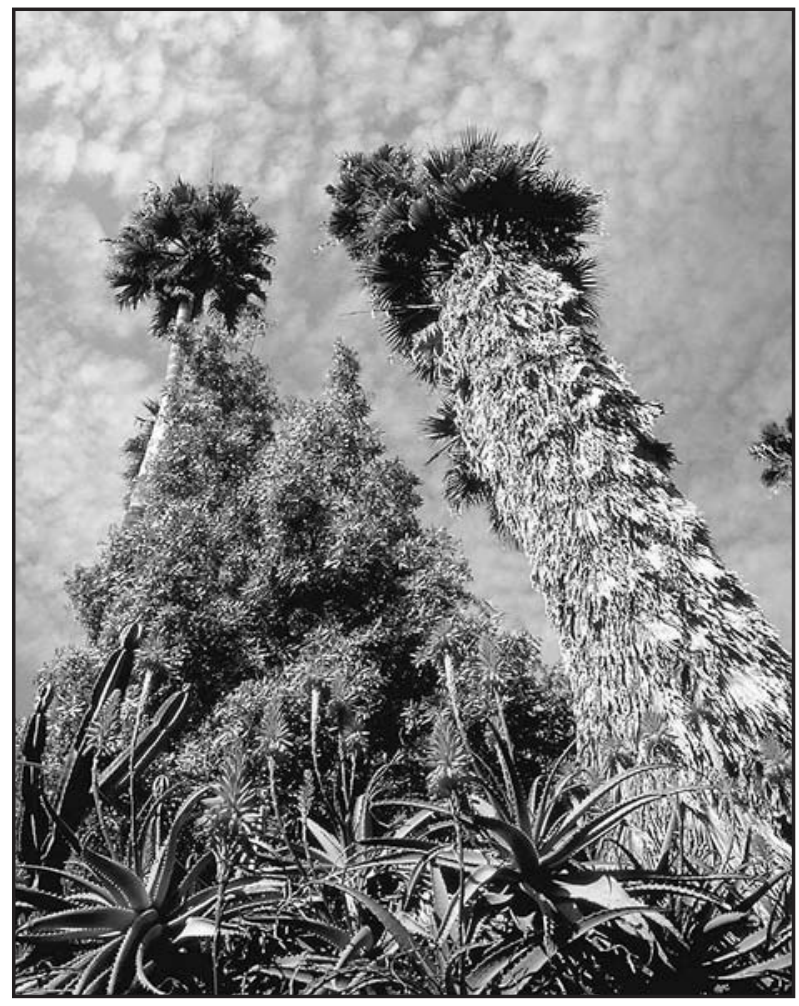

